\title{
Research on Mathematical Model for Non-Uniformly Qualitative Suspension Cable
}

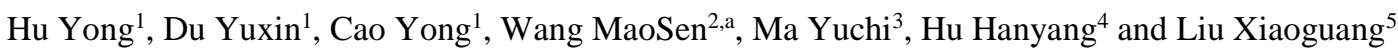 \\ ${ }^{1}$ School of Mechanical Science and Engineering, Jilin University, Changchun 130022, China \\ ${ }^{2}$ College of Construction Engineering, Jilin University, Changchun 130026, China \\ ${ }^{3}$ Ford Motor Research and Engineering (Nanjing) Co., Ltd, Nanjing 210000, China \\ ${ }^{4}$ College of Automotive Engineering, Jilin University, Changchun 130022, China \\ ${ }^{5}$ Aircraft and Dynamic Department, Aviation University of Air Force, Changchun 130022, China
}

\begin{abstract}
Catenary theory is recognized as the most effective suspension theory which can reflect the real situation of suspension. Catenary equation belongs to transcendental function, so there are some difficulties in calculation and application. Parabola theory, suspension curve theory and perturbation method are gradually formed as the theoretical basis for the study of suspension cables. This paper takes the $500 \mathrm{~m}$ spherical radio telescope project as the background. The modeling method of suspension cable under multiple concentrated loads is analyzed, and the mathematical model of supporting cable after load is derived. The research in this paper provides a reference for the establishment of mathematical model of suspension cable in practice.
\end{abstract}

\section{Introduction}

Five hundred meters Aperture Spherical Telescope (FAST) [1-2] is designed and built by our country. It is the world's largest single-caliber radio telescope and become the main unit of the international VLBI network.

When working, timely monitoring and feedback of the location and posture of the feed cabin are done by the cables including power cables and signal cables in the ground observation base. The traditional method of connecting cables into the cabin is to fix the cables on the rigid supports and reserve a certain working length. This length should guarantee the movement of cabin in its moving range. The connection between the feed cabin and the driving mechanism can only be flexible cables, which change the length and direction with the moving of the feed cabin. Therefore, the traditional method cannot meet requirements.

This paper research the homogeneous suspension catenary theory and parabola theory, and analysis and comparison,when the suspension by a single concentrated load is divided into two sections, the two sections of each suspension is consistent with the theory of suspension [3]. Based on the modeling method of the original multiple concentrated loads on the suspension, according to the structural characteristics, curtain mechanism supporting flexible feed system the radio telescope in the simplified model. the supporting cable is divided into three sections, each section of the concentrated load is approximately uniform load, piecewise mathematical model of the suspension.

\section{Mathematical models of suspension cable}

\subsection{Catenary and parabola}

In the engineering, steel cables are usually used as suspension cables. Suspended on two points, under the force of gravity, the cable has rigidity when a short straight segment is chosen to be studied. While it has flexibility when its span is large enough.

As shown in Figure 1, the suspension cable fixed at point $O$ and point A exhibits the shape of curve under the influence of gravity. Point $O$ is taken as the origin of coordinate, the $x$-axis is the horizontal line through the point $O$ and the $y$-axis is the vertical line through point $O$. The horizontal distance between point $O$ and point $A$ is $l$, and the vertical distance is $h$. The angle between the chord connecting point $O$ and point $A$ and the $x$-axis is $\alpha$. Point $D$ is the lowest point of the cable, of which the abscissa is $x_{\mathrm{d}}$.

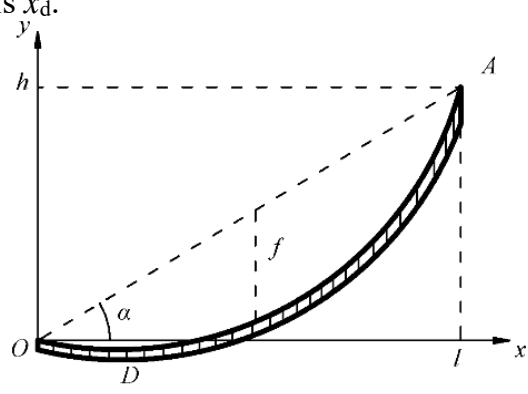

Figure 1. Curve of suspension cable $O A$

\footnotetext{
a Corresponding author: wangms@jlu.edu.cn
} 
The general equation of catenary [4] is

$$
y=(H / q) \operatorname{ch}\left[(q / H)\left(x+c_{1}\right)\right]+c_{2}
$$

where $c_{1}, c_{2}$ is arbitrary real. $H$ is the horizontal component of its tensile force and it can be proved that $H$ is a constant in a catenary. The length of catenary is

$$
L_{\text {oa }}=l \sec \alpha\left(1+\frac{8}{3} S^{2} \cos ^{4} \alpha\right)
$$

where $S=f_{0} / l, S$ called central deflection coefficient is the ratio of deflection to span and the barycentric coordinate can be deducted as

$$
\bar{x}=\frac{H}{q L_{\text {oa }}}\left(l \operatorname{sh} \frac{q}{H}\left(l-x_{\mathrm{d}}\right)-h\right)=\frac{l}{2}+\frac{h}{2 L_{\mathrm{oa}}}\left(\operatorname{lcth} \frac{q l}{2 H}-\frac{2 H}{q}\right)
$$

The tensile force of catenary is

$$
T=\frac{H}{\cos \beta}=H \operatorname{ch} \frac{q}{H}\left(x-x_{\mathrm{d}}\right)
$$

Talk about the parabola, suspended with point $O$ and point $A$, suspension cable assumes the shape shown in Figure 1 . The length of suspension cable is $L$, the gravity of per length is $q$. The tensile force of parabola is

$$
T=H \sqrt{1+\left(\tan \alpha-4 S\left(1-\frac{2 x}{l}\right)\right)^{2}}
$$

\subsection{Comparison and analysis of suspension theory}

The premise of both catenary theory and parabola theory is that the weight is uniformly distributed along the suspension cable. After mathematical derivation and for the drape curve shown in Figure 1, the boundary condition can be substituted. Then the equation (1) can be written as

$$
y=x \tan \alpha-\frac{q}{2 H}(l-x) x-\frac{\eta}{12 H}\left(l^{3}-x^{3}\right) x
$$

If the load ratio is too large, the action point of the moving concentrated load is from one end of the suspension to the other, and its trajectory is an ellipse with the focus on both ends. On the contrary, the linear shape of the suspension cable is almost unchanged, and its trajectory is approximately the same as that of the suspension curve without load.

By analyzing the above two suspensions, we can be concluded that when the suspension is divided into two sections by a single concentrated load, the two segments are still in line with the catenary model and the parabola model respectively.

\section{Simplification of curtain mechanism}

One suspension modeling method is to establish mathematical model according to suspension theory [4], the other is to model and combine according to the number of concentrated loads [5]. Combined with these two modeling methods, the supporting cable is divided into three sections according to the law of load distribution of curtain mechanism. The concentrated load on each segment is approximately equal to the uniform distribution load. The weight of the supporting cable is the weight of the unit length of the suspension cable. In each section of the suspension, the Cartesian coordinate system is established at the left end point and the segmented suspension model is derived according to the suspension theory. As shown in Figure 2.

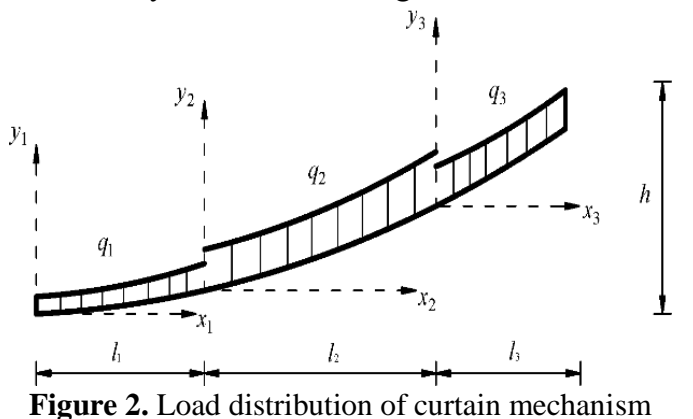

Set up the segmented catenary model. Mathematical model of each segment is established in turn based on the theory of suspension cable. The equation of segmented catenary is

$$
\left\{\begin{array}{l}
y_{i}(x)=\frac{2 H}{q_{i}} \operatorname{sh} \frac{q_{i}}{2 H}\left(x+2 c_{i}\right) \operatorname{sh} \frac{q_{i} x}{2 H} \\
c_{i}=-\frac{l_{i}}{2}+\frac{H}{q_{i}} \operatorname{arsh}\left(\frac{q_{i} h_{i}}{2 H} \operatorname{sh}^{-1} \frac{q_{i} l_{i}}{2 H}\right)
\end{array}\right.
$$

where $i=1,2,3 \ldots$ And the length of the suspension cable is

$$
L=\sum_{i=1}^{3} L_{i}=\sum_{i=1}^{3} \frac{1}{q_{i}} \sqrt{q_{i}^{2} h_{i}^{2}+4 H^{2} s h^{2} \frac{q_{i} l_{i}}{2 H}}
$$

And $c_{i}$ can be written as

$$
c_{i}=\frac{H}{q} \ln \frac{\frac{q_{i} h_{i}}{H}+\sqrt{\left(\frac{q_{i} h_{i}}{H}\right)^{2}+4 s h^{2} \frac{q_{i} l_{i}}{2 H}}}{e^{\frac{q_{i} l_{i}}{H}}-1}
$$

Equation (9) is parameter expression given by literature [6].

Set up the segmented parabolic model. Based on Equation (6) and take the left end of each segment as the origin of the coordinate system, the segmented parabolic model of the whole supporting cable is deduced as

$$
y_{i}(x)=x \tan \alpha_{i}-\frac{p_{i}}{2 H}\left(l_{i}-x\right) x
$$

where $i=1,2,3 \ldots$ And the length of the suspension cable is

$$
\left\{\begin{array}{l}
L=\sum_{i=1}^{3} L_{i}=l_{i} \sec \alpha_{i}\left(1+\frac{8}{3} S_{i}^{2} \cos ^{4} \alpha_{i}\right) \\
S_{i}=\frac{p_{i} l_{i}}{8 H}
\end{array}\right.
$$

\section{Simulation of suspension cable based on ADAMS}

\subsection{Modelling of bushing method}


With comparative analysis, the method of shaft sleeve force is used to model the wire rope. Different specifications of the wire rope can be simulated by setting two parameters in the bushing. The parameters are:

(1) Stiffness coefficient.

A steel wire rope is separated into several small cylinders. The length of the cylinder is $L$, the crosssectional area is $A$, the cross-section diameter is $D$, the moment of inertia is $I$, the shear modulus is $G$, the elastic modulus is $E$. There is no exact formula for the elastic modulus of the steel wire rope, which is related to its structure, the degree of wear, the length of the rope, load, tension and other factors.

As we know, according to the material mechanics, the stiffness coefficient is calculated as

$$
\left\{\begin{array}{l}
K_{11}=\frac{E A}{L} \\
K_{22}=K_{33}=\frac{G A}{L} \\
K_{44}=\frac{G \pi D^{4}}{32 L} \\
K_{55}=K_{66}=\frac{E I}{L}=\frac{E \pi D^{4}}{64 L}
\end{array}\right.
$$

(2) Damping coefficient.

In the literature [7], the influence of the damping coefficient on the mechanical properties of the flexible cable was studied by the experimental simulation. It reaches the conclusion that the tensile damping coefficient has little effect on the motion performance of the steel wire rope, and the torsional damping coefficient has obvious influence on that. The value is usually between $1 \sim 10 \mathrm{~N} \cdot \mathrm{s} / \mathrm{mm}$ for the situation.

After mathematical derivation, the reaction force of bushing is calculated as follows

$$
\left\{\begin{array}{l}
F_{j}=-F_{1} \\
T_{j}=-T_{i}-\delta \times F_{1}
\end{array}\right.
$$

\subsection{Simulation verification of wire rope}

The wire rope is modeled through the macro commands by using bushing method. The length of each cylinder is $L=30 \mathrm{~mm}$, the cross-sectional diameter is $D=\Phi 12 \mathrm{~mm}$, the elastic modulus is $E=207 \mathrm{GPa}$, the shear modulus $G=65.51 \mathrm{GPa}$, and the Poisson's ratio is $\mu=0.29$. The stiffness coefficient is calculated by Equation (12). According to the situation, the tensile damping coefficient takes $2 \mathrm{~N} \cdot \mathrm{s} / \mathrm{mm}$, and torsional damping coefficient takes $9 \mathrm{~N} \cdot \mathrm{s} / \mathrm{mm}$.

With the default integrator, the trajectory of the wire rope is simulated. The simulation time is $5 \mathrm{~s}$, and the number of simulation steps is 100 . The horizontal force of one end is simulated, shown in Figure 3. The force increases and reaches the peak within $0.5 \mathrm{~s}$, then gradually reduces and fluctuates, and finally stabilizes after $2 \mathrm{~s}$.

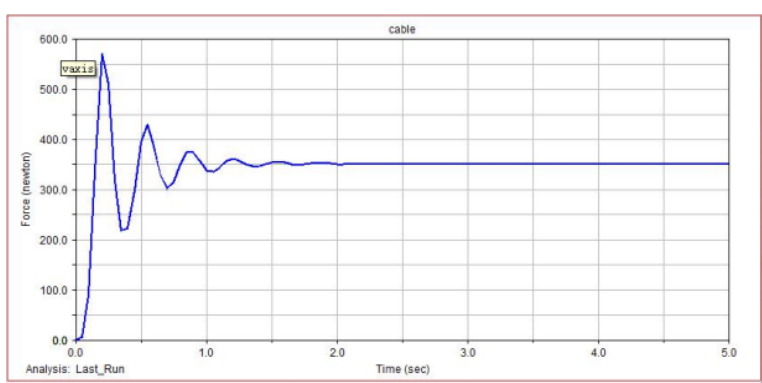

Figure 3. Horizontal force curve

The Cartesian coordinate system is established based on the left end of the suspension cable. The displacement of the point at the $1 / 4$ span of the suspension cable is measured in the $y$-axis. As shown in Figure 4, the displacement increases rapidly and reaches the peak within $0.4 \mathrm{~s}$, then decreases and fluctuates, and finally stabilizes after $2 \mathrm{~s}$. The negative sign indicates that the displacement direction is opposite to the $y$-axis direction.

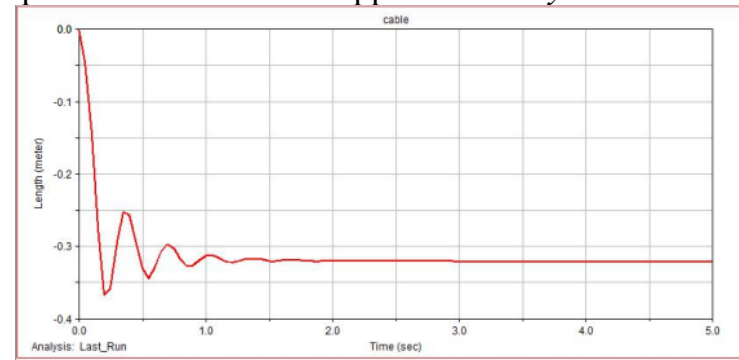

Figure 4. Displacement curve in y-axis direction

Similarly, the displacement in the $y$-axis direction of the suspension cable was measured at $2 / 5,1 / 2,3 / 5,3 / 4$, and these concentrated loads are converted into uniform load. The uniform load plus the weight of the suspension cable itself is the new weight of unit length Equation (14) is the mathematical model derived based on catenary theory. The corresponding $x$-axis coordinates of each point are substituted into Equation (14) to calculate the value of $y$, and the result is recorded in Table 1.

$$
y=\frac{H}{q}\left(\operatorname{ch} \frac{q}{H}\left(x-\frac{l}{2}\right)-\operatorname{ch} \frac{q l}{2 H}\right)
$$

Table 1. Displacement in $y$-axis direction for catenary model

\begin{tabular}{llllll}
\hline$y$ & $1 / 4$ & $2 / 5$ & $1 / 2$ & $3 / 5$ & $3 / 4$ \\
\hline $\begin{array}{l}\text { Simulation } \\
\text { value } / \mathrm{m}\end{array}$ & -0.319 & -0.415 & -0.463 & -0.444 & -0.335 \\
$\begin{array}{l}\text { Calculated } \\
\text { value } / \mathrm{m}\end{array}$ & -0.340 & -0.413 & -0.456 & -0.439 & -0.344 \\
$\begin{array}{l}\text { Relative } \\
\text { difference } / \%\end{array}$ & 6.58 & 0.48 & 1.51 & 1.12 & 2.68 \\
\hline
\end{tabular}

Equation (15) is the mathematical model derived based on parabolic theory. The corresponding $x$-axis coordinates of each point are substituted into Equation (14) to calculate the value of $y$, and the result is recorded in Table 2.

$$
y=\frac{q x}{2 H}(x-l)
$$

Table 2. Displacement in $x$-axis direction for catenary model 


\begin{tabular}{|c|c|c|c|c|c|}
\hline $\begin{array}{l}\mathrm{y} \\
\mathrm{x}\end{array}$ & $1 / 4$ & $2 / 5$ & $1 / 2$ & $3 / 5$ & $3 / 4$ \\
\hline $\begin{array}{l}\text { Simulation } \\
\text { value /m }\end{array}$ & -0.337 & -0.410 & -0.463 & -0.452 & -0.342 \\
\hline $\begin{array}{l}\text { Relative } \\
\text { difference } / \%\end{array}$ & 5.64 & 1.20 & 2.37 & 2.03 & 2.08 \\
\hline
\end{tabular}

Compared with Table 1 and Table 2, it can be concluded that both theoretical models can effectively describe the model of suspension cable, which verifies the feasibility of converting the concentrated loads into a uniform load. And the whole precision of catenary model is better than that of parabola model. The relative difference of the catenary model is less than that of the parabolic model, and a large error value appears near the end of the suspension. The main reason for this is that its weight is not sufficient to form a curve of catenary near the end of the suspension cable as the wire rope has rigidity.

\section{Conclusions}

In this paper, the mathematical model on non-uniformly qualitative suspension cable is studied by analyzing the mathematical model on uniformly qualitative suspension cable. By analyzing modeling method of the suspension cable with multiple concentrated loads, the curtain mechanism is simplified and segmented, then the mathematical model of the whole mechanism is deduced. Based on ADAMS virtual prototyping platform, the wire rope is simulated with existing modules and constraints. In this part, the method of virtual modeling is explored, and the feasibility of converting the concentrated loads into a uniform load is verified. At the end of this paper, the load ratio and the span of the supporting cable in the feed support system are selected as the single value conditions, and a steel wire rope model with a plurality of concentrated loads is built. In this paper, the curves fitting according to the measured values is compared with the models deduced from catenary theory and parabola theory, within three segments respectively. By analyzing the model error, it can be concluded that the catenary model can reflect the actual curve of suspension cable more accurately than the parabolic model.

\section{References}

1. Nan Rendong, $500 \mathrm{~m}$ spherical Reflector Radio Telescope FAST, Chinese Science $G$ Series of physical Mechanics Astronomy, 35(5), 449 (2005) (in Chinese)

2. Nan Rendong, Five hundred meter aperture spherical radio telescope(FAST), Science in China: Series G Physics, Mechanics \& Astronomy, 49(2), 129 (2006)

3. Kato Chengping, Design method of Forest aerial ropeway, Beijing: agricultural Publishing House (in Chinese)
4. Янпольский A.P, Translated by Xing Fuchong, Hyperbolic function, Beijing: central Institute of nationalities, Edition of the Society, (1987)

5. Zheng Lifeng, Theoretical derivation of multi-load catenary algorithm for single-span cableway, (Doctoral dissertation, Fujian Forestry University) (2002) (in Chinese)

6. Li Hui, Zhu Wenbai, Pan Gaofeng, Mechanics problems and research progress in feed support of FAST telescope, Mechanics Progress, 41(2),133 (2011) (in Chinese)

7. Chen Zili, Deng Ruiji, Principal Resonance Analysis of suspension Cable under concentrated load , Journal of Dynamics and Control, 5(4), 350 (2007) 